\title{
Chemical-Biology-derived in vivo Sensors: Past, Present, and Future
}

\author{
Robbie Loewith ${ }^{\star a b}$, Aurélien Roux ${ }^{\star a c}$, and Olivier Pertz ${ }^{\star d}$
}

\begin{abstract}
To understand the complex biochemistry and biophysics of biological systems, one needs to be able to monitor local concentrations of molecules, physical properties of macromolecular assemblies and activation status of signaling pathways, in real time, within single cells, and at high spatio-temporal resolution. Here we look at the tools that have been / are being / need to be provided by chemical biology to address these challenges. In particular, we highlight the utility of molecular probes that help to better measure mechanical forces and flux through key signalling pathways. Chemical biology can be used to both build biosensors to visualize, but also actuators to perturb biological processes. An emergent theme is the possibility to multiplex measurements of multiple cellular processes. Advances in microscopy automation now allow us to acquire datasets for 1000's of cells. This produces high dimensional datasets that require computer vision approaches that automate image analysis. The high dimensionality of these datasets are often not immediately accessible to human intuition, and, similarly to 'omics technologies, require statistical approaches for their exploitation. The field of biosensor imaging is therefore experiencing a multidisciplinary transition that will enable it to realize its full potential as a tool to provide a deeper appreciation of cell physiology.
\end{abstract}

Keywords: Biophysics · Biosensor · Machine learning · Signaling dynamics · Single cell biology

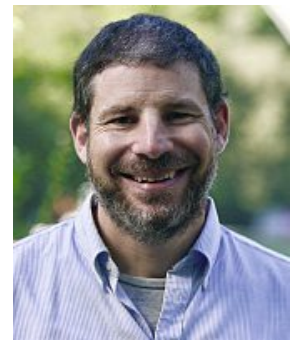

Robbie Loewith studied biochemistry, molecular biology and genetics at the University of Guelph, ON, Canada where he received his $\mathrm{BSc}$. For his $\mathrm{PhD}$, he moved to the University of Calgary, AB, Canada where he studied in the department of Biochemistry and Molecular Biology, graduating in 2000. He then moved to Basel, Switzerland, for post-doctoral work under the mentorship of Prof. Michael Hall. During this time he discovered that TOR (Target Of Rapamycin), a protein kinase that governs cell growth in virtually all eukaryotes, functions as part of two, distinct, multiprotein complexes. In 2005, still intrigued by TOR signalling, he started his own independent group at the University of Geneva where he is now a full professor in the Department of Molecular Biology and Director of the National Centre for Competence in Research in Chemical Biology (NCCR Chemical Biology). While rapamycin-sensitive TOR Complex 1 (TORC1) is well established to be regulated downstream of nutrient cues, the Loewith lab, in collaboration with the Roux lab, recently discovered that rapamycin-insensitive TORC2 functions in a homeostatic feedback loop to maintain tensile properties of the plasma membrane. Study of this unanticipated role of TORC 2 in mechanotransduction as well as determination of electron cryo-microscopy structures of the TOR complexes and their regulators are presently major foci of the Loewith group.

\footnotetext{
${ }^{\star}$ Correspondence: Prof. R. Loewith ${ }^{\text {ab }}$, Robbie.Loewith@unige.ch, Dr. A. Roux ${ }^{\text {ac }}$ Aurélien.Roux@unige.ch, Dr. O.Pertz ${ }^{\mathrm{d}}$, Olivier.Pertz@izb.unibe.ch aNational Centre for Competence in Research in Chemical Biology; bDepartment of Molecular Biology, University of Geneva;

'Department of Biochemistry, University of Geneva,

dnstitute of Cell Biology, University of Bern
}

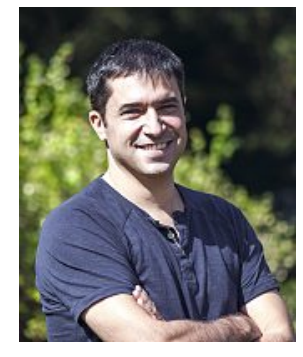

Aurélien Roux studied molecular biology at the Ecole Normale Supérieure de Lyon, France (BSc 2000) and physics at the Université Paris 6-Denis Diderot, Paris, France (MSc 2001, $\mathrm{PhD} 2004)$. He then moved to Yale for postdoctoral work under the mentorship of Pietro De Camilli. In 2007 he returned to Europe, first to the Institut Curie, Paris, France as a CNRS staff scientist and then, in 2010, to the University of Geneva where he is now a full professor in the department of Biochemistry. His lab aims to understand how the unique physical properties of lipid membranes and epithelia respectively contribute to membrane traffic and organogenesis. To these ends, they employ in vitro models to measure relevant physical parameters, such as forces, rigidity, tension, and diffusion constants, and in vivo biosensors to probe how membrane tension effects cellular signaling and membrane trafficking.

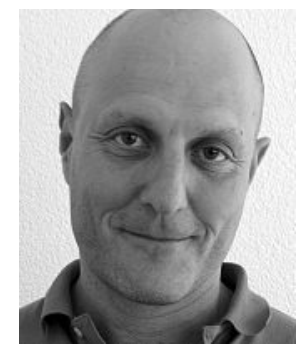

Olivier Pertz studied biology at the University of Lausanne and then biophysics at the University of Basel where he received his $\mathrm{PhD}$ in 1999. For his post-doctoral work he moved to the Scripps Research Institute, CA, USA, and in 2004 he became a Staff Scientist at the University of California San Diego, CA, USA. He then returned to the University of Basel as an Assistant professor in 2015 and since 2016 he is an Associate professor at the University of Bern in the Institute of Cell biology. His lab studies the spatio-temporal aspects of signaling events that regulate Cell Morphogenesis and Fate Decisions using novel quantitative approaches to measure/manipulate signaling dynamics at biologically relevant time/length scales.

\section{Brief Introduction}

The study of molecular cell biology has been aided tremendously by the development of chemical tools that label cellu- 
lar factors and/or alter their activities. Indeed, from vital stains and radio-labelled metabolites, to small-molecule agonists, antagonists and active-site and allosteric inhibitors, cell biology is built on the development of reagents with designed properties to measure and perturb activities of biomolecules with high spatiotemporal resolution. These chemistry-backed advances are often developed hand-in-hand with molecular biology tools, a classic example being the 'magic bullet for kinases' - the engineering of the 'gatekeeper' residue in the catalytic pocket as a generic approach to inhibit the enzymatic activity of virtually any protein kinase of interest. ${ }^{[1]}$ Subsequent examples include the generation of genetically-encoded, self-labeling protein tags such as the Halo, SNAP and CLIP tags, ${ }^{[2]}$ and rapamycin-based protein dimerization systems such as the anchor-away, ${ }^{[3]}$ or knock-sideways systems. ${ }^{[4]}$ Optogenetics ${ }^{[5]}$ represents an important variation on this chemical biology theme wherein cellular activities are manipulated by light rather than small molecules. Speaking of light, combining the now rich palette of fluorescent and bioluminescent proteins with fluorescent dyes incorporated onto self-labeling proteins offers opportunities to manipulate and monitor multiple processes simultaneously.

Figuratively and literally, the future looks bright. So what are the next challenges? As outlined below, many areas of cell biology are just now starting to benefit from the amazing power of chemical biology, while other areas still await the design of clever tools. We briefly review currently-available, state-of-the-art technologies, and provide some examples of how these tools have revolutionized our understanding of biology. Biosensor imaging now allows spectral multiplexing and is compatible with highthroughput imaging. This now produces high dimensional datasets analogous to 'omics methods. We discuss emerging computer vision approaches to analyze these large imaging datasets, and some of the statistical approaches to make sense of these complex data. By presenting recent innovative solutions, we hope that this review will inspire development of new tools to address currently difficult-to-access cell biology phenomena.

\section{What Can Be Sensed?}

There is now an incredible diversity of biosensors available and we make no attempt here to be comprehensive - rather, we begin with examples that typify what can be sensed and, by inference, what should be possible to sense in the near future.

\section{Sensing of Ions and Metabolites}

An early example of the power of biosensors was the development of $\mathrm{Ca}^{2+}$-sensitive dyes and genetically encoded calcium indicators. These tools have produced incredible images of $\mathrm{Ca}^{2+}$ 'puffs' and 'sparks' [6] in real-time with sub-micron resolution, and, combined with state-of-the-art microscopy techniques, have been used to monitor nerve firing in deep tissues. ${ }^{[7]}$ The latest variations include development of $\mathrm{pH}$-correctable $\mathrm{Ca}^{2+}$ sensors that enable accurate $\mathrm{Ca}^{2+}$ measurements in acidic organelles. ${ }^{[8]}$ $\mathrm{Ca}^{2+}$ sensing is reviewed extensively in the literature and not discussed further here; rather it serves as a high-water mark for what biosensors can achieve. Indeed, the remarkable successes in sensing $\mathrm{Ca}^{2+}$ inspired the creation of sensors of chloride, [9] $\mathrm{Cu}^{+[10]}$ as well as important metabolites such as $\mathrm{cAMP}{ }^{[11]}$ and nicotinamide adenine dinucleotides. ${ }^{[12]}$ Other analyte sensors are sure to follow. ${ }^{[13]}$

\section{Going beyond Sensing of Analytes}

Ion flux and metabolic conversions are clearly essential to life, but biological phenomena are also constrained by physics.

Although biochemical determinations are relatively accessible, assessing biophysical properties of cells continues to be much more challenging and represents an area where biosensors begin to have tremendous impact.

\section{Sensing of Molecular Crowding}

A good example of the sensing of a physical property is the sensing of molecular crowding. The past few years have seen an increasing appreciation for the importance of molecular crowding in a variety of cellular processes ranging from controlling the kinetics of biochemical reactions to regulating flux and dispersion of information. ${ }^{[14]}$ Specifically, formation of membraneless organelles by liquid-liquid phase separation (LLPS) is now realized to be a ubiquitous process to organize cellular content. LLPS is a dynamic process wherein proteins or nucleic acids self-assemble into amorphous structures that locally alter diffusion properties. LLPS of a given protein is relatively easy to follow with fluorescent tags although the fluorescent properties of these tags may be altered upon entry into the condensed state (where $\mathrm{pH}$ and physical proximity of fluorophores may lead to signal quenching) making quantitation challenging. LLPS of nucleic acids can be followed via a number of approaches including bisbenzimide dyes whose UV-induced fluorescence increases 30-fold upon binding to nucleic acid. Various fluorescence in situ hybridization techniques also exist. RNAs can also be detected in vivo by inserting sequences that are specifically recognized by RNA-binding proteins which themselves can be fluorescently labelled.

LLPS is triggered by several factors including REDOX, $\mathrm{pH}$, temperature, and, of course, cytoplasmic concentration. Cytoplasmic concentration or viscosity was recently shown to be regulated downstream of Target Of Rapamycin (TOR) signaling. ${ }^{[15]}$ To measure cytoplasmic viscosity, the authors used microrheology. Specifically, they expressed genetically encoded multimeric nanoparticles in cells and by single-molecule tracking using time-lapse fluorescence microscopy imaging, determined diffusion coefficients and derived the viscosity of the cytoplasm. By altering cytoplasmic crowding TOR could influence the LLPS of multiple factors. Intriguingly, cytoplasmic dynamics can also be manipulated experimentally. Focused-light-induced cytoplasmic streaming (FLUCS) uses a laser to induce hydrodynamic flows within the cytosplasm. ${ }^{[16]}$ With this technique, one can probe the functions of cytoplasmic flows and the material properties of structures such as centrosomes. The combination of FLUCS with microrheology and LLPS detection promises to yield exciting insights into the function of phase separation of specific factors.

\section{Sensing of Membrane Tension}

Lipid membranes have unique biophysical properties important for cell viability and dissecting how these various properties contribute to diverse cell functions has also become an important focus in cell biology. Lipid membranes are 2D fluids which can be deformed through stretching, causing tension, bending, causing curvature, and shearing, causing viscous flows. Thus, measuring tension, curvature and viscosity of lipid membranes is gaining increasing interest in cell biology. Owing to their hydrophobicity, lipid membranes are easy targets for appropriately-designed chemical compounds. Indeed, chemical biology approaches have provided new tools for measuring these properties. A good example is the ability to assess lipid order, the property of lipids to form domains of different phases, thereby segregating membrane components. ${ }^{[17]}$ Lipid order changes the hydration of lipids, which is then reported by shifts in the fluorescence emission spectra of Laurdan, a simple fluorescent molecule, and one of the first examples of a chemical probe sensitive to membrane biophysical properties. These molecules are solvatochromic push-pull probes, meaning that they have one electron-donor group, and one electron-receptor group. The efficiency of the electron transfer between the two groups affects fluorescence spectra, and depends on the solvation state of the molecule. ${ }^{[18]}$

More complex molecules are required to measure other biophysical parameters. Molecular rotors, which rotate with Brownian motion, can locally probe the mobility of molecules within the 
membrane, and are thus excellent reporters of membrane viscosity (or fluidity). ${ }^{[19]}$ In this case, the electron transfer between the fluorophore and the rest of the molecule depends on the speed at which the fluorophore rotates, which depends, in turn, on viscosity. Many fluorescent molecules can be used as molecular rotors, and, in many cases, the electron-transfer affects the fluorescence lifetime, which provides a useful way to do fluorescence measurements independent of the dye concentration.

Recently, molecular rotors have been proposed to report membrane tension, the surface force opposing membrane extension. ${ }^{[19]}$ But the most prominent advance in measuring membrane tension with fluorescent dyes has been made with the design of flipper molecules. ${ }^{[20]}$ Flippers are also push-pull probes, with electron transfer also affecting fluorescence lifetime. But instead of rotating, two planar fluorophores linked through a single carbon bond are kept out of planar alignment through electrostatic repulsion between additional groups. Mechanical pressure exerted by surrounding molecules - e.g. lipid acyl chains - can planarize the two fluorophores. Thus, flippers are real mechano-sensing molecules, directly reporting the pressure on their chemical structures. As membrane tension varies the pressure exerted on lipid acyl chains, flippers inserted in artificial and cellular membranes can report changes of tension through changes of their fluorescent lifetime.[21] However, for both rotors and flippers, fluorescence properties are strongly affected by lipid composition, lipid order, and phase segregation state of the membrane being probed.

It remains a challenge to measure membrane curvature with fluorescent membrane dyes. One feature of curvature that could be exploited for curvature sensing is the opposite tensions in the two leaflets of the curved membrane, one being stretched, the other one being compressed. However, as the sum of expected tension changes from both leaflets is null, curvature sensing would require leaflet-specific dye targeting. Also, curvature occurs on length scales much larger than the lipid/molecule size, which makes single lipids insensitive to curvature, ${ }^{[22]}$ but larger lipid domains, forming close to a demixing point, are sensitive to curvature.

\section{Sensing of Cytoskeletal Forces}

Beyond membrane tension, forces exerted by the cytoskeleton are also of strong interest to cell biologists. Chemical Biology tools that could measure forces along actin or microtubule filament lengths, or at their tips, would be extremely useful to help follow, for example, the force fields acting in lamellipodia of migrating cells, during the formation and the separation of the mitotic spindle, or during constriction of the contractile ring of the cleavage furrow during cytokinesis. One could imagine visualizing force fields in large cytoskeletal structures, and correlating this to the force fields with cell movements, or cell shape changes. One strategy could be to exploit chemical groups of natural products that specifically target filamentous forms of cytoskeletal proteins, such as jasplakinolide for actin-F, or taxol for tubulin. Linking two protomers with a biomechanical sensing spacer could report forces applied to neighbouring subunits within the filament structure. This could be a folded FRET reporter that unfolds upon stretch, or, a series of fluorophores and quenchers that change fluorescence upon compression. This would extend new possibilities to the field of mechanobiology, both at the single cell level, and at the tissue level.

\section{Sensing of Signaling Pathways}

Cells have developed highly specialized signaling networks to translate external (chemokines, mechanical cues, etc.) and internal (metabolic state, mechanical state, etc.) inputs into signaling outputs that specify fate decisions such as proliferation, death, survival or motility. An emerging picture is that signaling dynamics rather than static steady states regulate fate decisions. ${ }^{[23]}$ The present challenge is to understand how the feedback circuitry of signaling networks can produce rich sets of dynamic signaling behaviours. This is particularly important as corruption of the signaling flux through these pathways typically leads to grave diseases including metabolic syndrome and cancer. Biosensor imaging now provides a handle to study these signaling dynamics at the single cell level, at relevant time and length scales, in native and pathophysiological states.

A prime example of how biosensor imaging has revolutionized our understanding of the dynamic behaviour of a signaling pathway is the MAPK/ERK (Mitogen-activated protein kinase/ Extracellular signal-regulated kinase) pathway. The importance of ERK dynamics was initially hinted at by biochemical observations that different growth factors induce different ERK dynamics to control fates such as proliferation and differentiation. ${ }^{[24]}$ Since then, measurements of single-cell ERK dynamics using biosensors have revealed that a rich set of dynamic ERK activity patterns can emerge in different cell systems. These include transient, sustained, pulsatile and oscillatory ERK dynamics.[25] Heterogeneous signaling behaviours are typically observed within different cells of the population. These have been averaged out during classic population-average biochemical assays. At the cell population scale, collective behaviours such as waves of ERK pulses can be observed in epithelia during wound healing ${ }^{[26]}$ or apoptotic cell extrusion. ${ }^{[27]}$ The biosensor technology has therefore unveiled several complex behaviours that redefines the current MAPK signaling paradigms that have emerged using mostly classic biochemical approaches. Understanding this complex biology will require the complete arsenal of chemical biology-based biosensors and actuators to systematically measure and perturb different nodes of the MAPK network. These quantitative data will inform mathematical models to provide a true quantitative view of this pathway. Similar results are emerging for other signaling pathways.

Which chemical biology biosensors and actuators need to be built, and how can they be used to study a signaling network of choice? On one hand, we will need a large palette of biosensors to measure the dynamics of signal flux through multiple signaling nodes in response to standardized inputs. Chemical biology can provide the spectral flexibility to perform multiplexed measurements of multiple signaling activities simultaneously. For example, outside of the context of MAPK biology, simultaneous single-cell measurements of RhoA and Cdc42 GTPase activities were made possible by a combination of a genetically-encoded fluorescence resonance energy transfer-based reporter together with a cyanine-labeled domain-based biosensor. ${ }^{[28]}$ The realization that signaling networks fluctuate on timescales of minutes or seconds means that we need to rethink our traditional perturbation toolkit (drug, RNA interference, gene editing, etc.), as such longterm perturbations often lead to complete rewiring of the signaling system. Thus, true understanding of these temporal processes requires not only the ability to measure but also the ability to perturb them at these specific timescales. For example, temporally varying the signaling input, using microfluidics technology to challenge cells with growth factor pulses of varying concentration, duration or frequency, and systematically measuring the signaling output, coupled with mathematical modelling, has greatly refined our understanding of the MAPK network circuitry. ${ }^{[25 b]}$ Similar approaches using a combination of signaling optogenetics and biosensor imaging have provided higher throughput for measuring such signaling input-output responses. ${ }^{[29]}$ The existence of optogenetic actuators that can activate ERK at different levels within the MAPK network, e.g. RAS, ${ }^{[30]} \mathrm{RAF},{ }^{[31]}$ or MEK, ${ }^{[32]}$ provides opportunity to systematically map the flow of signaling in this cascade. Here again, a chemical biology approach can produce flexible perturbation tools, such as actuators or rapamycin-based knocksideways approaches, that can allow one to systematically 


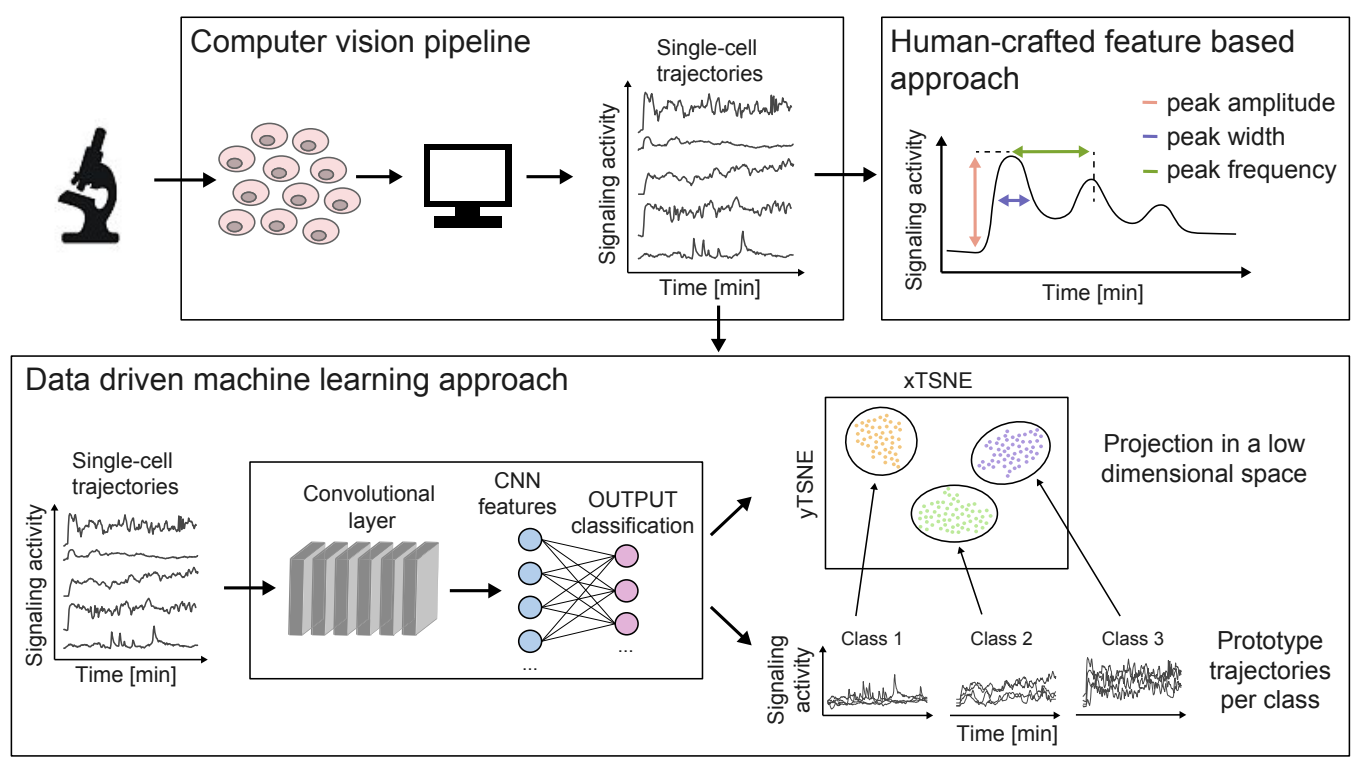

Fig. 1. Typical analysis pipeline for biosensor time-series datasets. Upper left panel. Images acquired using automated time-lapse microscopy are analyzed using a combination of image analysis pipelines, and yield large volumes of time-series datasets. One approach (upper right panel) involves human inspection of the time-series and formulation of a number of features that might capture different properties of the time-series. An advantage of this approach is that it is directly interpretable by human intuition. Another approach (lower panel) involves classification of time-series in response to different perturbations using convolutional neural networks (CNN). This produces a latent space of CNN features that can be projected in a low dimensional space that provides intuition about different classes (e.g. dynamic signaling behaviours). This can be used to extract prototype signaling trajectories relevant to a perturbation. This approach provides hypotheses about signaling behaviours, and is amenable to large datasets.

perturb signaling at relevant timescales. The fact that some of these chemical biology tools are 'dark', e.g. rapamycin-based systems, nicely complement multiplexed measurements/perturbations of fluorescence/optogenetics-based biosensors/actuator systems. Automation of the application of perturbations can be performed using microfluidic platforms that can be programmed to perform many experiments simultaneously or sequentially. ${ }^{[33]}$ Integration of chemical biology techniques with programmable microfluidics and automated imaging therefore can provide the ability to comprehensively perturb and measure biological systems at relevant time and length scales.

\section{Challenges Inherent to Large Biosensor Image Datasets}

The approaches mentioned above, when combined with automated microscopy, now provide the opportunity to image thousands of single cells in different perturbed states. This yields large datasets of similar or even larger complexity and size than sequencing-based bioinformatics. The emergent challenge now becomes the ability to effectively exploit these data. Many opensource image analysis platforms have recently emerged to tackle this issue. This includes packages such as Fiji, [34] CellProfiler, [35] and Ilastik ${ }^{[36]}$ to cite a few. Among many other tasks, these pipelines can perform segmentation and tracking of objects that allows for extraction of single-cell time-series. Machine learning approaches, based on user-annotated datasets, can segment specific regions of interest (ROIs) directly on images (for example nuclei). This bypasses classic 'threshold'-based segmentation approaches that typically do not perform well in low signal to noise images. In a setting in which a high volume of images must be analyzed, intensive computational resources might be required. A solution to this need is the usage of cloud-based computational resources such as Google Colab (https://colab.research.google. com). ZeroCostDL4MIC, for example, provides a framework to perform different deep learning-based image analysis tasks directly on Google Colab, without extensive need for programming expertise. ${ }^{[37]}$ This rapidly evolving portfolio of computational resources provides a low barrier access to image analysis accessible to people with little programming experience.
These image analysis pipelines provide the opportunity to extract the signaling time-series within thousands of single cells (or at specific subcellular locations within single cells). Very often, averages of the intrinsically heterogeneous signaling activities within a population are still computed to generate initial intuition about signaling networks. The caveat of this approach however is that it does not resolve subtle signaling patterns that arise due to extrinsic or intrinsic noise within biochemical pathways - essential information when dissecting such signaling pathways. While bioinformatics pipelines to analyze single-cell RNAseq data are now mainstream, such pipelines are just emerging in the field of single-cell biosensor time-series. One approach to mine signaling trajectories datasets requires initial human evaluation of the signaling trajectories and crafting of human-intuitive features that captures properties from the signaling time-series (Fig. 1). For example, in a dataset of pulsatile ERK activity, this could capture the amplitude, the width, and frequency of ERK pulses. ${ }^{[38]}$ Plotting these features can then provide intuition regarding the different finite signaling states within cells of a population. However, having to browse through many single-cell trajectories, in response to different perturbations, can quickly prove overwhelming to human intuition. A complementary, purely data-driven approach therefore consists of using convolutional neuronal networks (CNN) to identify patterns in signaling time-series in different perturbed states (Fig. 1). ${ }^{[39]}$ This approach can then identify prototype signaling trajectories that capture the most discriminative features relevant to a signaling state, which are often different than the population average. Another advantage of this approach is that it allows to project the signaling trajectories in a low dimensional space that provides intuition about the heterogeneity and similarity of different single-cell signaling states in response to different perturbations. This provides a new way to interact with high dimensional datasets signaling time-series enabling intuitive exploration of large datasets and thus hypotheses about the underlying biology. This approach has revealed the existence of a variety of ERK/AKT single-cell activity dynamics in response to a large variety of growth factors that signal through different receptor tyrosine kinases. ${ }^{[39]}$ These examples showcase how scalable chemical biology-based biosensor and actuators, when interfaced with 
modern imaging, image and statistical analysis pipelines, will be able to comprehensively explore signaling networks.

\section{Conclusions}

Chemical Biology has made huge advances in enabling scientists to monitor critical biochemical parameters in vivo and in real time. Excitingly, next generation biosensors are now coming online to monitor critical biophysical parameters, although the need for new tools in this domain remains. The ability to perform multiplexed measurements in response to perturbations applied at adequate time and length scales have the potential to highly enhance our understanding of cellular processes. Biosensor imaging produces increasingly complex high dimensional datasets as complex as those obtained with 'omics approaches. Computational methods are now also being produced for such kinds of datasets. The analysis of these cellular processes, at adequate spatial and temporal scales, and at scale will provide the quantitative data for a more exhaustive understanding of cellular processes, which are also amenable to mathematical modelling. The future will see probes that work in tissues, organs and even individuals.

\section{Acknowledgements}

This work was supported by grants from the Swiss National Science Foundation (RL, OP, AR), the NCCR Chemical Biology (RL, AR), Human Frontier Science Program (OP), and the Swiss Cancer League (OP). We are grateful to Coralie Dessauges for help producing Fig. 1.

Received: October 27, 2021

[1] A. C. Bishop, O. Buzko, K. M. Shokat, Trends Cell Biol. 2001, 11, 167.

[2] J. Wilhelm, S. Kuhn, M. Tarnawski, G. Gotthard, J. Tunnermann, T. Tanzer, J. Karpenko, N. Mertes, L. Xue, U. Uhrig, J. Reinstein, J. Hiblot, K. Johnsson, Biochemistry 2021, 60, 2560, https://doi.org/10.1021/acs.biochem.1c00258.

[3] H. Haruki, J. Nishikawa, U. K. Laemmli, Mol. Cell 2008, 31, 925, https://doi.org/10.1016/j.molcel.2008.07.020.

[4] M. S. Robinson, D. A. Sahlender, S. D. Foster, Dev. Cell 2010, 18, 324, https://doi.org/10.1016/j.devcel.2009.12.015.

[5] C. P. O'Banion, D. S. Lawrence, ChemBioChem 2018, 19, 1201, https://doi.org/10.1002/cbic.201800013.

[6] https://www.youtube.com/watch?v=7LyZkNeyw9s

[7] W. Yang, R. Yuste, Nat. Methods 2017, 14, 349, https://doi.org/10.1038/nmeth.4230.

[8] N. Narayanaswamy, K. Chakraborty, A. Saminathan, E. Zeichner, K. Leung, J. Devany, Y. Krishnan, Nat. Methods 2019, 16, 95, https://doi.org/10.1038/s41592-018-0232-7.

[9] M. Zajac, K. Chakraborty, S. Saha, V. Mahadevan, D. T. Infield, A. Accardi, Z. Qiu, Y. Krishnan, J. Cell Sci. 2020, 133, https://doi.org/10.1242/jcs.240390.

[10] M. Priessner, P. A. Summers, B. W. Lewis, M. Sastre, L. Ying, M. K. Kuimova, R. Vilar, Angew. Chem. Int. Ed. 2021, 60, 23148, https://doi.org/10.1002/anie.202109349.

[11] N. Kim, S. Shin, S. W. Bae, Biosensors 2021, 11, https://doi.org/10.3390/bios11020039.

[12] O. Sallin, L. Reymond, C. Gondrand, F. Raith, B. Koch, K. Johnsson, eLife 2018, 7, https://doi.org/10.7554/eLife.32638.

[13] J. A. Kaczmarski, J. A. Mitchell, M. A. Spence, V. Vongsouthi, C. J. Jackson, Curr. Opin. Struct. Biol. 2019, 57, 31, https://doi.org/10.1016/j.sbi.2019.01.013.

[14] M. Hondele, S. Heinrich, P. De Los Rios, K. Weis, Emer. Top. Life Sci. 2020 4, 331, https://doi.org/10.1042/ETLS20190190.

[15] M. Delarue, G. P. Brittingham, S. Pfeffer, I. V. Surovtsev, S. Pinglay, K. J. Kennedy, M. Schaffer, J. I. Gutierrez, D. Sang, G. Poterewicz, J. K. Chung, J. M. Plitzko, J. T. Groves, C. Jacobs-Wagner, B. D. Engel, L. J. Holt, Cell 2018, 174, 338, https://doi.org/10.1016/j.cell.2018.05.042.

[16] a) M. Mittasch, P. Gross, M. Nestler, A. W. Fritsch, C. Iserman, M. Kar, M. Munder, A. Voigt, S. Alberti, S. W. Grill, M. Kreysing, Nat. Cell Biol. 2018, 20, 344, https://doi.org/10.1038/s41556-017-0032-9; b) M Mittasch, V. M. Tran, M. U. Rios, A. W. Fritsch, S. J. Enos, B. Ferreira Gomes, A. Bond, M. Kreysing, J. B. Woodruff, J. Cell Biol. 2020, 219, https://doi.org/10.1083/jcb.201912036.

[17] A. S. Klymchenko, Acc. Chem. Res. 2017, 50, 366, https://doi.org/10.1021/acs.accounts.6b00517.

[18] Y. Niko, A. S. Klymchenko, J. Biochem. 2021, 170, 163 , https://doi.org/10.1093/jb/mvab078.
[19] M. Paez-Perez, I. Lopez-Duarte, A. Vysniauskas, N. J. Brooks, M. K. Kuimova, Chem. Sci. 2020, 12, 2604, https://doi.org/10.1039/d0sc05874b.

[20] a) M. Dal Molin, Q. Verolet, A. Colom, R. Letrun, E. Derivery, M. GonzalezGaitan, E. Vauthey, A. Roux, N. Sakai, S. Matile, J. Am. Chem. Soc. 2015 137, 568, https://doi.org/10.1021/ja5107018; b) L. Assies, J. García-Calvo, F. Piazzolla, S. Sanchez, T. Kato, L. Reymond, A. Goujon, A. Colom, J. López-Andarias, K. Straková, D. Mahecic, V. Mercier, M. Riggi, N. JiménezRojo, C. Roffay, G. Licari, M. Tsemperouli, F. Neuhaus, A. Fürstenberg, E. Vauthey, S. Hoogendoorn, M. Gonzalez-Gaitan, A. Zumbuehl, K. Sugihara, J. Gruenberg, H. Riezman, R. Loewith, S. Manley, A. Roux, N. Winssinger, N. Sakai, S. Pitsch, S. Matile, CHIMIA 2021, 75, 1004, https://doi.org/10.2533/chimia.2021.1004.

[21] A. Colom, E. Derivery, S. Soleimanpour, C. Tomba, M. Dal Molin, N. Sakai, M. González-Gaitán, S. Matile, A. Roux, Nat. Chem. 2018, in press, https://doi.org/10.1038/s41557-018-0127-3.

[22] B. Sorre, PhD Thesis, 2010, http://www.theses.fr/2010PA077021.

[23] J. E. Purvis, G. Lahav, Cell 2013, 152, 945, https://doi.org/10.1016/j.cell.2013.02.005.

[24] R. Avraham, Y. Yarden, Nat. Rev. Mol. Cell Biol. 2011, 12, 104 https://doi.org/10.1038/nrm3048.

[25] a) J. G. Albeck, G. B. Mills, J. S. Brugge, Mol. Cell 2013, 49, 249, https://doi.org/10.1016/j.molcel.2012.11.002; b) Y. Blum, J. Mikelson, M. Dobrzynski, H. Ryu, M. A. Jacques, N. L. Jeon, M. Khammash, O. Pertz, Mol. Syst. Biol. 2019, 15, e8947, https://doi.org/10.15252/msb.20198947.

[26] N. Hino, L. Rossetti, A. Marin-Llaurado, K. Aoki, X. Trepat, M. Matsuda, T. Hirashima, Dev. Cell 2020, 53, 646, https://doi.org/10.1016/j.devcel.2020.05.011

[27] P. A. Gagliardi, M. Dobrzynski, M. A. Jacques, C. Dessauges, P. Ender, Y. Blum, R. M. Hughes, A. R. Cohen, O. Pertz, Dev. Cell 2021, 56, 1712, https://doi.org/10.1016/j.devcel.2021.05.007.

[28] M. Machacek, L. Hodgson, C. Welch, H. Elliott, O. Pertz, P. Nalbant, A Abell, G. L. Johnson, K. M. Hahn, G. Danuser, Nature 2009, 461, 99, https://doi.org/10.1038/nature08242.

[29] C. Dessauges, J. Mikelson, M. Dobrzynski, M.-A. Jacques, A Frismantiene, P. A. Gagliardi, M. Khammash, O. Pertz, bioRxiv 2021 https://doi.org/10.1101/2021.07.27.453955.

[30] M. Z. Wilson, P. T. Ravindran, W. A. Lim, J. E. Toettcher, Mol. Cell 2017, 67, 757, https://doi.org/10.1016/j.molcel.2017.07.016.

[31] K. Aoki, Y. Kumagai, A. Sakurai, N. Komatsu, Y. Fujita, C. Shionyu, M. Matsuda, Mol. Cell 2013, 52, 529, https://doi.org/10.1016/j.molcel.2013.09.015.

[32] A. L. Patel, E. Yeung, S. E. McGuire, A. Y. Wu, J. E. Toettcher, R. D. Burdine, S. Y. Shvartsman, Proc. Natl. Acad. Sci. USA 2019, 116, 25756, https://doi.org/10.1073/pnas.1912320116.

[33] S. Tay, J. J. Hughey, T. K. Lee, T. Lipniacki, S. R. Quake, M. W. Covert, Nature 2010, 466, 267, https://doi.org/10.1038/nature09145.

[34] J. Schindelin, I. Arganda-Carreras, E. Frise, V. Kaynig, M. Longair, T. Pietzsch, S. Preibisch, C. Rueden, S. Saalfeld, B. Schmid, J. Y. Tinevez, D. J. White, V. Hartenstein, K. Eliceiri, P. Tomancak, A. Cardona, Nat. Methods 2012, 9, 676, https://doi.org/10.1038/nmeth.2019.

[35] L. Kamentsky, T. R. Jones, A. Fraser, M. A. Bray, D. J. Logan, K. L. Madden, V. Ljosa, C. Rueden, K. W. Eliceiri, A. E. Carpenter, Bioinformatics 2011, 27, 1179, https://doi.org/10.1093/bioinformatics/btr095.

[36] S. Berg, D. Kutra, T. Kroeger, C. N. Straehle, B. X. Kausler, C. Haubold, M. Schiegg, J. Ales, T. Beier, M. Rudy, K. Eren, J. I. Cervantes, B. Xu, F. Beuttenmueller, A. Wolny, C. Zhang, U. Koethe, F. A. Hamprecht, A. Kreshuk, Nat. Methods 2019, 16, 1226 https://doi.org/10.1038/s41592-019-0582-9.

[37] L. von Chamier, R. F. Laine, J. Jukkala, C. Spahn, D. Krentzel, E. Nehme, M. Lerche, S. Hernandez-Perez, P. K. Mattila, E. Karinou, S. Holden, A. C. Solak, A. Krull, T. O. Buchholz, M. L. Jones, L. A. Royer, C. Leterrier Y. Shechtman, F. Jug, M. Heilemann, G. Jacquemet, R. Henriques, Nat. Commun. 2021, 12, 2276, https://doi.org/10.1038/s41467-021-22518-0.

[38] T. E. Gillies, M. Pargett, M. Minguet, A. E. Davies, J. G. Albeck, Cell Syst. 2017, 5, 549, https://doi.org/10.1016/j.cels.2017.10.019.

[39] M. A. Jacques, M. Dobrzynski, P. A. Gagliardi, R. Sznitman, O. Pertz, Mol. Syst. Biol. 2021, 17, e10026, https://doi.org/10.15252/msb.202010026.

\section{License and Terms}

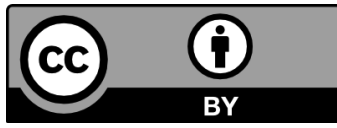

This is an Open Access article under the terms of the Creative Commons Attribution License CC BY 4.0. The material may not be used for commercial purposes.

The license is subject to the CHIMIA terms and conditions: (http:// chimia.ch/component/sppagebuilder/?view=page \&id=12).

The definitive version of this article is the electronic one that can be found at https://doi.org/10.2533/chimia.2021.1017 